\title{
CURVATURE AND THE EIGENVALUES OF THE LAPLACIAN
}

\author{
H. P. MCKEAN, JR. \& I. M. SINGER
}

\section{Introduction}

A famous formula of $H$. Weyl [19] states that if $D$ is a bounded region of $R^{d}$ with a piecewise smooth boundary $B$, and if $0>\gamma_{1} \geq \gamma_{2} \geq \gamma_{3} \geq$ etc. $\downarrow-\infty$ is the spectrum of the problem

$$
\begin{gathered}
\Delta f=\left(\partial^{2} / \partial x_{1}^{2}+\cdots+\partial^{2} / \partial x_{d}^{2}\right) f=\gamma f \text { in } D, \\
f \in C^{2}(D) \cap C(\bar{D}), \\
f=0 \text { on } B
\end{gathered}
$$

then

$$
-\gamma_{n} \sim C(d)(n / \operatorname{vol} D)^{2 / d}(n \uparrow \infty)
$$

or, what is the same,

$$
Z \equiv \operatorname{sp} e^{l \Delta}=\sum_{n \geq 1} \exp \left(\gamma_{n} t\right) \sim(4 \pi t)^{-d / 2} \times \operatorname{vol} D(t \downarrow 0)
$$

where $C(d)=2 \pi[d / 2) !]^{d / 2}$.

$\AA$. Pleijel [13] and $M$. Kac [6] took up the matter of finding corrections to (3) for plane regions $D$ with a finite number of holes. The problem is to find how the spectrum of $\Delta$ reflects the shape of $D$. Kac puts things in the following amusing language : thinking of $D$ as a drum and $0<-\gamma_{1}<-\gamma_{2} \leq$ etc. as its fundamental tones, is it possible, just by listening with a perfect ear, to hear the shape of $D$ ? Weyl's estimate (2) shows that you can hear the area of $D$. Kac proved that for $D$ bounded by a broken line $B$,

$$
\text { (4a) } \begin{aligned}
Z= & \frac{\text { area } D}{4 \pi t}-\frac{\text { length } B / 4}{\sqrt{4 \pi t}} \\
& + \text { the sum over the corners of } \frac{\pi^{2}-\gamma^{2}}{24 \pi \gamma}+o(1) \quad(t \downarrow 0),
\end{aligned}
$$
dation under NSF GP-4364 and NSF GP-6166 is gratefully acknowledged. 
$0<\gamma<2 \pi$ being the inside-facing angle at the corner ${ }^{1}$, esp., you can hear the perimeter of such $D$. By making the broken line $B$ approximate to a smooth curve, Kac was led to conjecture

$$
Z=\frac{\text { area }}{4 \pi t}-\frac{\text { length } / 4}{\sqrt{4 \pi t}}+\frac{1}{6}(1-h)+o(1) \quad(t \downarrow 0)
$$

for regions $D$ with smooth $B$ and $h<\infty$ holes, and was able to prove the correctness of the first 2 terms. This jibes with an earlier conjecture of $\AA$. Pleijel and suggests that you can hear the number of holes. (4b) will be proved below in a form applicable both to open manifolds with compact boundary and to closed manifolds.

Given a closed $d$-dimensional, smooth Riemannian manifold $M$ with metric tensor $g=\left(g_{i j}\right)$, let $\Delta$ be the associated Laplace-Beltrami operator:

$$
\Delta=\frac{1}{\sqrt{\operatorname{det} g}} \frac{\partial}{\partial x_{i}} g^{i j} \sqrt{\operatorname{det} g} \frac{\partial}{\partial x_{j}}
$$

where $g^{-1}=\left(g^{i j}\right)$, and let $0=\gamma_{0}>\gamma_{1} \geq \gamma_{2} \geq$ etc. $\downarrow-\infty$ be its spectrum. Define also the scalar curvature $K$ at a point of $M$ (= the negative of the spur $\sum_{i<j} R_{i j}^{i j}$ of the Ricci tensor) and partition function $Z \equiv \operatorname{sp} e^{i \Delta}=\sum \exp \left(\gamma_{n} t\right)$. Then, as will be proved in $\S \S 4$ and 7 ,

$$
\begin{aligned}
& (4 \pi t)^{d / 2} Z=\text { the (Riemannian) volume of } M \\
& +\frac{t}{3} \times \text { the curvatura integra } \int_{M} K+\frac{t^{2}}{180} \int_{M}(10 A-B+2 C)+o\left(t^{3}\right),
\end{aligned}
$$

where $\int_{M}$ stands for the integral relative to the Riemannian volume element $\sqrt{\operatorname{det} g} d x$, and $A, B, C$ stand for a particular basis of the space of polynomials of degree 2 in the curvature tensor $R$ which are invariant under the action of the orthogonal group [see (7.2)]; $0\left(t^{3}\right)$ cannot be improved. For $d=2,10 A-B+2 C=12 K^{2}$, and an application of the classical Gauss-Bonnet formula for the Euler characteristic $E$ of $M\left(2 \pi E=\int_{M} K\right),(5 a)$ simplifies to

${ }^{1} \mathrm{Kac}$ [6] expresses the corner correction $\left(\pi^{2}-\gamma^{2}\right) / 24 \pi \gamma$ as complicated integral. D. B. Ray [private communication] derived it by a simpler argument, beginning with the Green function $G$ for $s-\Delta(s>0)$ expressed as a Kantorovich-Lebedev transform

$$
\begin{aligned}
G(A, B) & =\pi^{-2} \int_{0}^{\infty} d x K_{\sqrt{ }-1 x}(\sqrt{s} a) K_{\sqrt{ }-1 x}(\sqrt{s} b) \\
& \times\left[\cosh (\pi-|\alpha-\beta|) x-\frac{\sinh \pi x}{\sinh \gamma x} \cosh (\gamma-\alpha-\beta) x+\frac{\sinh (\pi-\gamma) x}{\sinh \gamma x} \cosh (\alpha-\beta) x\right],
\end{aligned}
$$

in which $A=a e \sqrt{-1} \alpha, B=b e \sqrt{-1} \beta$, and $K$ is the usual modified Bessel function. The corner correction $\left(\pi^{2}-\gamma^{2}\right) / 24 \pi \gamma$ follows easily, and this jibes with Kac's integral upon applying Parseval's formula to the latter. 


$$
Z=\frac{\text { area }}{4 \pi t}+\frac{E}{6}+\frac{\pi t}{60} \int_{M} K^{2}+o\left(t^{2}\right)
$$

esp., the Euler characteristic of $M$ is audible.

Consider now an open $d$-dimensional manifold $D$ with compact $(d$ 1)- dimensional boundary $B, \bar{D}=D \cup B$ being endowed with a smooth Riemannian geometry, and let $0>\gamma_{1}^{-} \geq \gamma_{2}^{-} \geq$etc. $\downarrow-\infty$ and $0=$ $\gamma_{0}^{+}>\gamma_{1}^{+} \geq \gamma_{2}^{+} \geq$etc. $\downarrow-\infty$ be the spectra of

$$
\begin{aligned}
& \Delta^{-}=\Delta \mid C^{\infty}(\bar{D}) \cap(u: u=0 \text { on } B) \\
& \Delta^{+}=\Delta \mid C^{\infty}(\bar{D}) \cap(u: u=0 \text { on } B)
\end{aligned}
$$

where $\cdot$ stands for differentiation in the inward-pointing direction perpendicular to $B$.

Bring in also the mean curvature $J$ at a point of $B(=$ double the spur of the second fundamental form) and the partition function $Z^{ \pm} \equiv$ $\operatorname{sp} e^{t \Delta \pm}=\sum \exp \left(\gamma_{n}^{ \pm} t\right)$. Then, as will be proved in $\S 5$,

$$
\begin{aligned}
& (4 \pi t)^{d / 2} Z^{ \pm}=\text {the (Riemannian) volume of } D \\
& \pm \frac{1}{4} \sqrt{4 \pi t} \times \text { the (Riemannian) surface area of } B \\
& +\frac{t}{3} \times \text { the curvatura integra } \int_{D} K \\
& -\frac{t}{6} \times \text { the integrated mean curvature } \int_{B} J+o\left(t^{3 / 2}\right)
\end{aligned}
$$

where $\int_{B}$ stands for the integral over $B$ relative to the element of Riemannian surface area ; $0\left(t^{3 / 2}\right)$ cannot be improved. Kac-Pleijel's conjecture (4b) for a plane region $D$ with smooth boundary $B$ and $h<\infty$ holes is obtained from (6) and the Gauss-Bonnet formula $\left(\int_{M} K+\int_{B} J=\right.$ $2 \pi \times$ the Euler characteristic) for the closed manifold $M=$ the double of $D$ upon noting that the Euler characteristic of the handle-body $M$ is just $2(1-h)$.

The estimates leading to (5) and (6) will be proved not just for $\Delta$ but for any smooth elliptic partial differential operator of degree $2(2,3$, $4,5)$, and some additional comments will be made about $Z=\operatorname{sp} e^{t \Delta}$ for $\Delta$ acting on exterior differential forms (6). The basic idea, due to Kac, is to make a pointwise estimate of the pole of the elementary solution of $\partial u / \partial t=\Delta u$ and then to integrate over $M$ to get an estimate of $Z=$ $\operatorname{sp} e^{t \Delta}$. The curvatura integra coefficient in (5a) is computed directly in $\S 4$ and then re-computed (for $\Delta$ only) in $\S 7$ using more sophisticated algebraic ideas about differential invariants of the orthogonal group. A list of open problems is placed at the end of the paper [9]. 
The new results of this paper are mainly for the case of manifolds with boundary. For a closed manifold, N. G. de Bruijn [private communication] obtained the curvatura integra coefficient independently as did V. Arnold [private communication from M. Berger]. Berger also kindly communicated his formula for the next coefficient, which suggested the approach in $\S 7$. Berger's results for closed manifolds can be found in [1]. His method is different from ours, but we arrive at the same formula for the coefficient of $t^{2}$ provided his norms $\tau^{2},|\rho|^{2}$, and $|R|^{2}$ are equal to our $4 A, B$, and $2 C$ respectively.

It is a pleasant duty to thank M. Kac for suggesting this problem and for a number of stimulating conversations about it. Thanks are also due to T. Kotake for help with the Levi sums of $\S 3$.

\section{Manifolds and elliptic operators}

Consider a closed, $d$-dimensional, smooth manifold $M$ and let $Q: C^{\infty}(M) \rightarrow$ $C^{\infty}(M)$ be an elliptic partial differential operator of degree 2, with $Q(1)=0$. On a patch $U \subset M, Q$ can be expressed as

$$
Q=a^{i j} \partial^{2} / \partial x_{i} \partial x_{j}+b^{i} \partial / \partial x_{i} \equiv a \partial^{2}+b \partial
$$

with coefficients $a=\left(a^{i j}\right)$ and $b=\left(b^{i}\right)$ from $C^{\infty}(U)$. By changing the sign of $Q$ if necessary, we can take the quadratic form based upon $a$ as positive $\left(\sum a^{i j} y_{i} y_{j}>0, y \neq 0\right)$, and under a change of local coordinates $x \rightarrow \bar{x}$ with Jacobian $c, a$ transforms according to the rule $\bar{a}=c a c^{*}$, so $g=a^{-1}$ transforms like a Riemannian metric tensor. $M$ is now endowed with this Riemannian geometry, and $Q$ is re-expressed as the sum of the associated Laplace-Beltrami operator $\Delta$ plus a part of degree 1:

$$
Q=\Delta+h \partial, h \partial=h^{i} \frac{\partial}{\partial x_{i}}, \quad \Delta=-\frac{1}{\sqrt{\operatorname{det} g} \partial x_{i}} g^{i j} \sqrt{\operatorname{det} g} \frac{\partial}{\partial x_{j}} .
$$

Because $\Delta$ does not depend upon the choice of local coordinates, $h \partial$ is a vector field.

$\Delta$ is symmetric $\left(\int u \Delta v=\int v \Delta u\right)$ and non-positive $\left(\int u \Delta u \leq 0\right)$ relative to the Riemannian volume element $\sqrt{\operatorname{det} g} d x$, where $\int f=\int_{M} f$ always means $\int_{M} f \sqrt{\operatorname{det} g} d x$. $Q$ enjoys the same properties relative to some volume element $e^{w} \sqrt{\operatorname{det} g} d x$ if and only if the vector field $h \partial$ is conservative; this is the same as to say that the exterior differential 1 -form dual to this field is an exact differential $(=d w)$, as is plain from the fact that, for a patch $U$ and compact $u$ and $v \epsilon C^{\infty}(U)$,

$$
\int_{U}(u Q v-v Q u) e^{w}=\int_{U}(u \operatorname{grad} v-v \operatorname{grad} u)\left(h-g^{-1} \operatorname{grad} w\right)
$$


cannot vanish unless $h=g^{-1} \operatorname{grad} w$ (Nelson [12]), where grad = $\left(\partial / \partial x_{1}, \cdots, \partial / \partial x_{d}\right)$.

Consider, next, the elementary solution $e=e(t, x, y)$ of $\partial u / \partial t=$ $Q u$ computed relative to the volume element $\sqrt{\operatorname{det} g} d x$ and recall the following facts:

$$
\begin{gathered}
0<e \in C^{\infty}\left[(0, \infty) \times M^{2}\right], \\
\partial e / \partial t=Q_{x} e=Q_{y}^{*} e, \\
\int e \sqrt{\operatorname{det} g} d y=1, \\
\lim _{t \downarrow 0} t^{-1} 1 g e=-\frac{1}{4}[x y]^{2}
\end{gathered}
$$

where $Q^{*}$ is the dual of $Q$ relative to $\sqrt{\operatorname{det} g} d x$, and $[x y]$ is the Riemannian distance between $x$ and $y$; see [16] for $(d)$ and [10] for the rest.

Now if $Q$ is symmetric relative to the volume element $e^{w} \sqrt{\operatorname{det} g} d x$, then $e(t, x, y) \exp [-w(y)]$ is symmetric in $x$ and $y$, and since its spur $Z=\int e(t, x, x)$ converges, $e^{t Q}: f \rightarrow \int e f$ is a compact mapping of the (real) Hilbert space $H=L^{2}\left[M, e^{w} \sqrt{\operatorname{det} g} d x\right]$. This implies that $Q$ has a discrete spectrum

$$
0=\gamma_{0}>\gamma_{1} \geq \gamma_{2} \geq \text { etc. } \uparrow-\infty
$$

with corresponding eigen functions $f_{n} \in C^{\infty}(M)$ forming a unit perpendicular basis of $H$; in addition,

$$
e=\sum_{n \geq 0} \exp \left(\gamma_{n} t\right) f_{n} \otimes f_{n}
$$

with uniform convergence on compact figures of $(0, \infty) \times M^{2}$, and the spur $Z$ is easily evaluated as (see for example [10])

$$
Z=\int \sum_{n \geq 0} \exp \left(\gamma_{n} t\right) f_{n}^{2} e^{w}=\sum_{n \geq 0} \exp \left(\gamma_{n} t\right) .
$$

Kac's method for the proof (4a) is now imitated to obtain (5a): one estimates the pole $e(t, x, x)$ locally and then integrates over $M$. This is done in $\S \S 3$ and 4 using a method of E. E. Levi; the actual estimate is just as easy for the general $Q$, so the condition that the vector field $h \partial$ be conservative is not insisted upon.

Now let $Q=\Delta+h \partial$ be defined on a smooth open, $d$-dimensional manifold $D$ with smooth, compact, $(d-1)$-dimensional boundary $B$, suppose that $g=a^{-1}$ is positive and smooth on the whole of $\bar{D}$ so that it induces a nice Riemannian geometry on $\bar{D}$, and let the vector field $h \partial$ be smooth on $\bar{D}$ too. Both $Q^{-}=Q \mid C^{\infty}(\bar{D}) \cap(u: u=0$ on $B)$ and $Q^{+}=Q \mid C^{\infty}(D) \cap\left(u: u^{\cdot}=0\right.$ on $\left.B\right)$, standing for differentiation in 
the inward-pointing direction perpendicular to $B$, have nice elementary solutions $e=e^{ \pm}$subject to

$$
0 \leq e \in C^{\infty}\left[(0, \infty) \times \bar{D}^{2}\right]
$$

$$
\frac{\partial e}{\partial t}=Q_{x} e=Q_{y}^{*} e
$$

$Q^{*}$ being the dual of $Q$ relative to $\sqrt{\operatorname{det} g} d x$,

$$
\begin{array}{cc}
(4 \mathrm{c}-) & \int_{D} e^{-} \downarrow 1 \quad(t \downarrow 0), \\
(4 \mathrm{c}+) & \int_{D} e^{+}=1, \\
(4 \mathrm{~d}) & \varlimsup_{t \downarrow 0} t^{-1} \lg e \leq-\frac{1}{4}[x y]^{2}, \\
(4 \mathrm{e}-) & e^{-}=0 \text { on } B \times D, \\
(4 \mathrm{e}+) & e^{+\cdot}=0 \text { on } B \times D,
\end{array}
$$

and for $Q$ symmetric relative to some volume element, the spectra are as before except at the upper end:

$$
\begin{gathered}
0>\gamma_{1}^{-} \geq \gamma_{2}^{-} \geq \text {etc. } \downarrow-\infty, \\
0=\gamma_{0}^{+}>\gamma_{1}^{+} \geq \gamma_{2}^{+} \text {etc. } \downarrow-\infty,
\end{gathered}
$$

and the formula for the partition function still holds:

$$
Z^{ \pm}=\int_{D} e^{ \pm}(t, x, x)=\sum \exp \left(\gamma_{n} t\right)
$$

so that (6) can likewise be derived by estimating the pole $e^{ \pm}(t, x, x)$.

\section{Levi's sum for the elementary solution}

Given closed $M$ and $Q=\Delta+h \partial$ as above, one can express the elementary solution $e=e(t, x, y)$ of $\partial u / \partial t=Q u$ by means of a sum due to E. $\mathrm{E}$. Levi; this computation has been carried out in a very careful manner by S. Minakshisundaram [10], but it will be helpful to indicate the idea in a form suited to the present use.

Consider a little closed patch $U$ of $M$ with smooth ( $d-1)$-dimensional boundary $B$, view $U$ as part of $R^{d}$, extend $Q^{\prime}=Q \mid U$ to the whole of $R^{d}$ in such a way that the coefficients of the extension belong to $C^{\infty}\left(R^{d}\right)$ and $Q^{\prime}=\partial^{2} / \partial x_{1}^{2}+\cdots+\partial^{2} / \partial x_{d}^{2}$ near $\infty$, let $e^{\prime}$ be the elementary solution of $\partial u / \partial t=Q^{\prime} u$, and let us prove that inside $U \times U$,

$$
\left|e^{\prime}-e\right| \leq \exp (\text {-constant } / t) \quad(t \downarrow 0)
$$


with a positive constant depending only upon the distance to $B$.

Proof. Bring in the elementary solution $e^{\prime \prime}$ of $\partial u / \partial t=Q u$ subject to $u=0$ on $B$. Given a compact function $v \in C^{\infty}(U), u=\int\left(e^{\prime \prime}-e\right) v$ solves $\partial u / \partial t=Q u$ on $(0, \infty) \times U$ and tends to 0 uniformly on $\bar{U}$ as $t \downarrow 0$. But this means that in the figure $[0, t] \times \bar{U},|u|$ peaks on $[0, t] \times B$, so that by an application of the estimate of Varadhan $[(2.1 \mathrm{~d}),(2.4 \mathrm{~d})]^{1}$,

$$
|u| \leq \max _{[0, t] \times B}\left|\int\left(e^{\prime \prime}-e\right) v\right| \leq \exp \left(-R^{2} / 5 t\right)\|v\|_{1},
$$

$R$ being the shortest (Riemannian) distance from $(v \neq 0) \subset U$ to $B$. The rest of the proof is self-evident.

Because of (1), it is permissible, for the estimation of the pole $e(t, x, x)$ up to an exponentially small error, to replace $M$ by $R^{d}$ and to suppose that $Q=\partial^{2} / \partial x_{1}^{2}+\cdots+\partial^{2} / \partial x_{d}^{2}$ far out; this modification of the problem is now adopted,

Define now $Q^{0}$ to be $Q$ with its coefficients frozen at $y \in R^{d}$, and let $e^{0}(t, x, y)$ be the elementary solution of $\partial u / \partial t=Q^{0} u$ evaluated at $t>0, x \in R^{d}$, and the same point $y \in R^{d}$ at which the coefficients of $Q^{0}$ are computed:

$$
e^{0}(t, x, y)=(4 \pi t)^{-d / 2} \exp \left(-\left|a^{0 \frac{1}{2}}\left(y-x-b^{0} t\right)\right|^{2} / 4 t\right)
$$

with an obvious notation. Because of (2. 1b), (2. 1c) and (2. 1d),

$$
\begin{aligned}
& e(t, x, y)-e^{0}(t, x, y)=\int_{0}^{t} d s \frac{\partial}{\partial s} \int_{R^{d}} e(s, x, \cdot) e^{0}(t-s, \cdot, y) \\
& =\int_{0}^{t} d s \int_{R^{d}}\left(e^{0} Q^{*} e-e Q^{0} e^{0}\right) \\
& =\int_{0}^{t} d s \int_{R^{d}} e(s, x, \cdot)\left(Q-Q^{0}\right) e^{0}(t-s, \cdot, y)
\end{aligned}
$$

in short,

$$
e=e^{0}+e \sharp f,
$$

with $\sharp$ denoting the composition on the final line of (3a) and

$$
f=\left(Q-Q^{0}\right) e^{0}(t-s, x, y)
$$

Upon iteration, this identity produces the (formal) sum for $e$ :

$$
e=e^{0}+\sum_{n \geq 1} e^{0} \sharp f \sharp \cdots \sharp f(n \text {-fold }) \text {. }
$$

\footnotetext{
${ }^{1}(2.1 \mathrm{~d})$ denotes equation (1d) of $\S 2$.
} 
Actually this formal sum converges to $e$ uniformly on compact figures of $(0, \infty) \times R^{2 d}$; the main point is that since

$$
Q=\partial^{2} / \partial x_{1}^{2}+\cdots+\partial^{2} / \partial x_{d}^{2} \text { near } \infty
$$

$$
\begin{aligned}
|f| & \leq c_{1}\left(\frac{|x-y|^{3}}{t^{2}}+\frac{|x-y|}{t}+1\right) t^{-d / 2} \exp \left(-c_{2}|x-y|^{2} / t\right) \\
& \leq c_{3} t^{-(d+1) / 2} \exp \left(-c_{4}|x-y|^{2} / t\right)
\end{aligned}
$$

$c_{1}, \cdots, c_{4}$ standing for positive constants, as can easily be verified by a direct computation, and this leads easily to the bound

$$
\left|e^{0} \sharp f \sharp \cdots \sharp f\right| \leq c_{5}^{n}[(n / 2) !]^{-1} t^{(n-d) / 2} \exp \left(-c_{6}|x-y|^{2} / t\right) .
$$

Accordingly, the formal sum (4) converges rapidly to a nice function $e$ of magnitude

$$
|e| \leq \sum_{n \geq 0} \frac{\left(c_{5} \sqrt{t}\right)^{n}}{(n / 2) !} t^{-d / 2} \exp \left(-c_{6}|x-y|^{2} / t\right)
$$

which satisfies (3b). A moment's reflection shows that $e$ is an elementary solution of $\partial u / \partial t=Q u$. But $\partial u / \partial t=Q u$ has only 1 elementary solution subject to $(6)$, so $e=(4)$ is it. This is proved by noticing that any elementary solution subject to $(6)$ is also a solution of $(3 \mathrm{~b})$, and then proving that $(3 \mathrm{~b})+(6)$ has just 1 solution.

\section{Estimation of the pole}

Levi's sum (3.4) can now be used to estimate the pole $e(t, x, x)$ for $t \downarrow 0$, up to terms of magnitude $t^{1-d / 2}$ :

$$
(4 \pi t)^{d / 2} e(t, x, x)=1+\frac{t}{3} K-\frac{t}{2} \operatorname{div} h-\frac{t}{4}|h|^{2}+0\left(t^{2}\right)
$$

in which $K$ is the scalar curvature (= the negative spur $\sum_{i<j} R_{i j}^{i j}$ of the

Riccitensor), $\operatorname{div} h$ is the (Riemannian) divergence $\left[=(\operatorname{det} g)^{-\frac{1}{2}} \partial h^{i}(\operatorname{det} g)^{\frac{1}{2}} / \partial x_{i}\right]$, and $|h|$ is the (Riemannian) length $\left(=g_{i j} h^{i} h^{j}\right)$. (1) can be integrated over $M$ to get an estimate of $Z=\int e(t, x, x)$ (since $\left.\int \operatorname{div} h=0\right)$ :

$$
(4 \pi t)^{d / 2} Z=\int 1+\frac{t}{3} \int K-\frac{t}{4} \int|h|^{2}+0\left(t^{2}\right)
$$

$e s p$. , if $Q=\Delta$, then $h=0$ and $(2)=(1.5 \mathrm{a})$. A little extra attention to the proof, which is left to the industrious reader, shows the existence of an expansion 


$$
(4 \pi t)^{d / 2} e(t, x, x)=1+k_{1} t+k_{2} t^{2}+\cdots+k_{n} t^{n}+o\left(t^{n+1}\right) .
$$

This was proved by S. Minakshisundaram [10] for $Q=\Delta$; the only novel point is the evaluation $k_{1}=K / 3-(\operatorname{div} h) / 2-|h|^{2} / 4 . k_{2}$ is computed in $\S 7$, using a more sophisticated method.

Proof of (1). $e$ can be replaced by the sum (3.4), and the terms of index $n \geq 4$ can be neglected in view of (3. 5b). Put $x=0$ for simplicity and bring in new coordinates on $R^{d}$ coinciding with the old near $\infty$ and such that

$$
g_{i j}(x)=\delta_{i j}+\frac{1}{3} R_{i k j l} x_{k} x_{l}+0\left(|x|^{3}\right) \quad \text { near } \quad o,
$$

$R$ being the curvature tensor associated with $g$; this is accomplished by applying the exponential map to the tangent space at 0 to obtain coordinates on a patch and then fixing things up outside [3, Chapter 10]. An estimate of $f=\left(Q-Q^{0}\right) e^{0}(t-s, x, y)$ finer than (3. 5a) is now possible:

$$
\begin{aligned}
& |f(t, x, y)| \leq c_{1}\left(\frac{|x||y-x|^{3}}{t^{2}}\right. \\
& \left.\quad+\frac{|x||y-x|}{t}+1\right) \exp \left(-c_{2}|x-y|^{2} / t\right)
\end{aligned}
$$

where $c_{1}, c_{2}$, etc. stand for positive constants. This is used to prove

$$
\begin{aligned}
\left|e^{0} \sharp f \sharp f\right| \leq & \int_{0}^{t} d s_{1} \int_{0}^{t} d s_{2} \int_{R^{2 d}} \frac{c_{3} e^{-c_{4}|x|^{2} /\left(t-s_{1}\right)}}{\left(t-s_{1}\right)^{d / 2}} \\
& \times\left(\frac{|x||y-x|^{3}}{t^{2}}+\frac{|x||y-x|}{t}+1\right) \frac{e^{-c_{4}|x-y|^{2} /\left(s_{1}-s_{2}\right)}}{\left(s_{1}-s_{2}\right)^{d / 2}} \\
& \times\left(\frac{|y|^{4}}{t^{2}}+\frac{|y|^{2}}{t}+1\right) \frac{e^{-c_{4}|y|^{2} / s_{2}}}{s_{2}^{d / 2}} \\
\leq & c_{5} t^{-d / 2} \int_{0}^{t} d s_{1} \int_{0}^{s_{1}} d s_{2} \sqrt{\frac{t-s_{1}}{s_{1}-s_{2}}}=c_{6} t^{2-d / 2}
\end{aligned}
$$

and the similar but easier bound

$$
\left|e^{0_{\sharp}} f \sharp f \sharp f\right| \leq c_{7} t^{2-d / 2},
$$

which shows that, up to terms of magnitude $\leq$ constant $\times t^{2-d / 2}$, one is left with

$$
\begin{aligned}
& e(t, 0,0)=e^{0}(t, 0,0) \\
& \quad+\int_{0}^{t} d s \int_{R^{d}} e^{0}(t-s, 0, x)\left(Q-Q^{0}\right) e^{0}(s, x, 0) \sqrt{\operatorname{det} g} d x
\end{aligned}
$$


A moment's reflection will convince the reader that, up to the desired precision, the integrand $e^{0}(t-s, 0, x)\left(Q-Q^{0}\right) e^{0}(s, x, 0) \sqrt{\operatorname{det} g}$ can be replaced by the product of a factor $1+$ a linear function $f$ of $x+o(t)+$ $o\left(|x|^{2}\right)$ and the expression

(8)

$$
\begin{aligned}
& \frac{e^{-|x|^{2} / 4(t-s)}}{[4 \pi(t-s)]^{d / 2}}\left[\frac{1}{2} \frac{\partial^{2} g^{i j}}{\partial x_{k} \partial x_{l}}(0) x_{k} x_{l} \frac{\partial^{2}}{\partial x_{i} \partial x_{j}}\right. \\
& +\left(\frac{\partial}{\partial x_{k}} \frac{1}{\sqrt{\operatorname{det} g}} \frac{\partial}{\partial x_{i}} g^{i j} \sqrt{\operatorname{det} g}\right)(0) x_{k} \frac{\partial}{\partial x_{j}} \\
& \left.+\frac{\partial h^{i}}{\partial x_{k}}(0) x_{k} \frac{\partial}{\partial x_{i}}\right] \frac{e^{-|x|^{2} / 4 s}}{(4 \pi s)^{d / 2}} \\
& =(4 \pi t)^{-d / 2} \frac{e^{-|x|^{2} / 4 r}}{(4 \pi r)^{d / 2}}\left[\frac{1}{2} \frac{\partial^{2} g^{i j}}{\partial x_{k} \partial x_{l}}(0) x_{k} x_{l}\left(\frac{x_{i} x_{j}}{4 s^{2}}-\frac{\delta_{i j}}{2 s}\right)\right. \\
& \left.-\left(\frac{\partial}{\partial x_{k}} \frac{1}{\sqrt{\operatorname{det} g}} \frac{\partial}{\partial x_{i}} g^{i j} \sqrt{\operatorname{det} g}\right)(0) \frac{x_{k} x_{j}}{2 s}-\frac{\partial h^{i}}{\partial x_{k}}(0) \frac{x_{i} x_{k}}{2 s}\right] \text {, }
\end{aligned}
$$

where $r=s(t-s) / t$. Now the factor alluded to above (8) can be replaced by 1 , since $f \times(8)$ integrates to 0 while the last 2 terms contribute $\leq c_{8} t^{2-d / 2}$. Consequently, up to the desired precision,

(9a)

$$
\begin{aligned}
& t^{-1}(4 \pi t)^{d / 2} e^{0} \sharp f=t^{-1} \int_{0}^{t} d s \int_{R^{d}}(8) d x \\
& =\frac{1}{2} \frac{\partial^{2} g^{i j}}{\partial x_{k} \partial x_{l}}(0) \times(0,1 / 3 \text {, or } 1 \text { according as } i j k l \text { comprises } \\
& \leq 1 \text { pair, } 2 \text { unequal pairs, or } 2 \text { equal pairs) } \\
& -\frac{1}{4} \frac{\partial^{2} g^{i j}}{\partial x_{k} \partial x_{l}}(0) \delta_{k l} \delta_{i j} \\
& -\frac{1}{2}\left(\frac{\partial}{\partial x_{k}} \frac{1}{\sqrt{\operatorname{det} g}} \frac{\partial}{\partial x_{i}} g^{i j} \sqrt{\operatorname{det} g}\right)(0) \delta_{k j}-\frac{1}{2} \frac{\partial h^{i}}{\partial x_{k}}(0) \delta_{k i} \\
& =\frac{1}{6} \frac{\partial^{2} g^{i i}}{\partial x_{j}^{2}}+\frac{1}{3} \frac{\partial^{2} g^{i j}}{\partial x_{i} d x_{j}}(\text { summed for } i \neq j)+\frac{1}{2} \frac{\partial^{2} g^{i i}}{\partial x_{i}^{2}} \\
& -\frac{1}{4} \frac{\partial^{2} g^{i i}}{\partial x_{j}^{2}}-\frac{1}{2} \frac{\partial}{\partial x_{j}} \frac{1}{\sqrt{\operatorname{det} g}} \frac{\partial}{\partial x_{i}} g^{i j} \sqrt{\operatorname{det} g}-\frac{1}{2} \frac{\partial h^{i}}{\partial x_{i}}, \\
& \text { all evaluated at } x=0 \text {. }
\end{aligned}
$$

Cartan's formula (4), combined with the skew symmetry of the curvature tensor $R$, permits an additional simplification of (9a) to 


$$
\begin{gathered}
-\frac{1}{6} \frac{\partial^{2} g^{i j}}{\partial x_{i} \partial x_{j}}-\frac{1}{2} \frac{\partial^{2} \sqrt{\operatorname{det} g}}{\partial x_{i}^{2}}-\frac{1}{12} \frac{\partial^{2} g^{i i}}{\partial x_{j}^{2}}-\frac{1}{2} \operatorname{div} h \\
=-\frac{1}{18} R_{i j i j}-\frac{1}{6} R_{i j i j}+\frac{1}{18} R_{i j i j}-\frac{1}{2} \operatorname{div} h \\
=-\frac{1}{3} \sum_{i<j} R_{i j i j}-\frac{1}{2} \operatorname{div} h=\frac{K}{3}-\frac{1}{2} \operatorname{div} h
\end{gathered}
$$

and (1) follows upon noting that

$$
(4 \pi t)^{d / 2} e^{0}(t, 0,0)=e^{-|h(0) t|^{2} / 4 t}=1-\frac{t}{4}|h|^{2}+o(t)^{2}
$$

\section{Manifolds with boundary}

Now let $D$ be an open manifold with compact boundary $B$ as at the end of $\S 2, M=D \cup B \cup D^{*}$ the (closed) double of $D$, and $Q$ the double to $M$ of a smooth elliptic operator of degree 2 on $D$, and, as in $\S 2$, define $Q^{-}\left(Q^{+}\right)$to be $Q \mid C^{\infty}(\bar{D})$ subject to $u=0(u=0)$ on $B$. The coefficients $(\operatorname{det} g)^{-\frac{1}{2}} \partial g^{i j}(\operatorname{det} g)^{\frac{1}{2}} / \partial x_{i}$ occurring in $Q$ jump as $x$ crosses $B$, but $\partial u / \partial t=Q u$ still has a nice elementary solution $e$ of class $C^{\infty}\left[(0, \infty) \times(M-B)^{2}\right] \cap C^{1}\left(M^{2}\right)$, approximable even on $B$ by Levi's sum, and the elementary solutions $e^{ \pm}$of $\partial u / \partial t=Q^{ \pm} u$ can be expressed on $(0, \infty) \times D^{2}$ as

$$
e^{ \pm}(t, x, y)=e(t, x, y) \pm e(t, x, \stackrel{*}{y})
$$

$\stackrel{*}{y} \in D^{*}$ being the double of $y \in D$. By use of this formula, $Z^{ \pm}=$ $\int_{D} e^{ \pm}(t, x, x)$ can be estimated as follows:

(2) $(4 \pi t)^{d / 2} Z^{ \pm}=$the (Riemannian) volume $\int_{D} 1$

$\pm \frac{1}{4} \sqrt{4 \pi t} \times$ the $\left(\right.$ Riemannian) surface area $\int_{B} 1$

$\pm \frac{t}{2} \int_{B}$ flux $h+\frac{t}{3} \times$ the curvatura integra $\int_{D} K$

$-\frac{t}{6} \times$ the integrated mean curvature $\int_{B} J$

$-\frac{t}{2} \int_{D} \operatorname{div} h-\frac{t}{4} \int_{D}|h|^{2}+o\left(t^{3 / 2}\right)$.

To explain the new terms involved in this formula, pick a self-double patch $U$ of $M$ covering a patch $U \cap B$ of $B$ endowed as in the diagram with local coordinates $x$ such that 


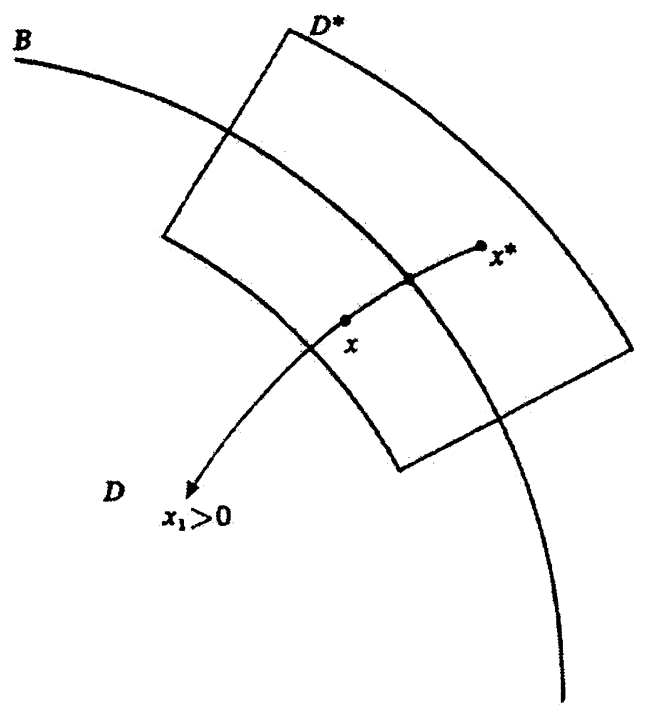

a) $1>x_{1}>0$ in $U \cap D$, b) $x_{1}=0$ on $U \cap B$, c) $x_{1}\left(x^{*}\right)=-x_{1}(x)$, and d) the positive $x_{1}$-direction is perpendicular to $B$. This has the effect that

$$
\begin{aligned}
g_{i j}\left(x^{*}\right) & =-g_{i j}(x) \text { for } i=1<j \text { or } i>j=1 \\
& =+g_{i j}(x) \text { for } i=j=1 \text { or } i, j \geq 2, \\
g_{i j}(x) & =0 \text { for } i=1<j \text { or } i>j=1 \text { on } B
\end{aligned}
$$

$\sqrt{\operatorname{det} g / g_{11}} d x_{2} \cdots d x_{d}=$ the element of (Riemannian) surface area on $B$.

Now $\int_{B}$ stands for integration relative to $\sqrt{\operatorname{det} g / g_{11}} d x_{2} \cdots d x_{d}$, flux $h$ is the (outward-pointing) flux of $h$ at a point of $B\left(=-\sqrt{g_{11}} h^{1}\right)$, and the mean curvature $J$ at a point of $B$ is (double) the spur of the second fundamental form $\left[=\left(g^{11} \operatorname{det} g\right)^{\cdot} \sqrt{g_{11}} / \operatorname{det} g\right]^{1}$, representing (twice) the sum of inner curvatures of 2-dimensional sections perpendicular to $B$. Because of Green's formula $\left(\int_{D} \operatorname{div} h=\int_{B}\right.$ flux $\left.h\right)$, a little cancellation

${ }^{1}$ Here ${ }^{\cdot}$ stands for the one-sided partial in the positive 1-direction perpendicular to $B$. To prove that $\left(g^{11} \operatorname{det} g\right)^{\cdot} \sqrt{g_{11}} / \operatorname{det} g$ is (double) the spur of the second fundamental form of $B$, it is preferable to further specialize the local coordinates on $U$ so as to make

$$
g=\left(\begin{array}{r}
g_{11} \mid 0 \\
0 \mid h
\end{array}\right) \text { on } U \text { and } g_{11}=1 \text { on } U \cap B
$$

The second fundamental form $f$ is the (Riemannian) gradient along $B$ of the inwardpointing unit normal field $n$ :

$$
f_{i j}=\frac{\partial n_{i}}{\partial x_{j}}+\left\{\begin{array}{c}
i \\
j k
\end{array}\right\} n_{k}=\left\{\begin{array}{c}
i \\
1 j
\end{array}\right\}=\text { the Christoffel bracket }(i, j \geq 2)
$$

Computing this for the special $g$ adopted above gives $\frac{1}{2} h^{-1} h^{\prime}$, so that double the spur is

$$
\operatorname{sp} h^{-1} h^{\cdot}=(1 \mathrm{~g} \operatorname{det} h)^{\cdot}=\left(1 \mathrm{~g} g^{11} \operatorname{det} g\right)^{\cdot}=\left(g^{11} \operatorname{det} g\right)^{\cdot} / \operatorname{det} g,
$$

as desired $\left(g^{11}=g_{11}=1\right.$ on $\left.B\right)$. 
occurs in (2) for $Q^{+} .(2)=(1.6)$ for $Q=\Delta(h=0)$. The proof of $(2)$ is broken up into a number of steps.

Step 1. Consider a subregion $D^{\prime} \subset D$ at a positive distance from B. Varadhan's bound $(2.4 \mathrm{~d})$ implies that $\int_{D^{\prime}} e(t, x, \dot{x}) \leq \exp \left(-c_{1} / t\right)$, so by $(4.1)$,

$$
\begin{aligned}
(4 \pi t)^{d / 2} \int_{D^{\prime}} e^{ \pm}(t, x, x)=\int_{D^{\prime}} & {\left[1+\frac{t}{3} K-\frac{t}{2} \operatorname{div} h-\frac{t}{4}|h|^{2}\right] } \\
& + \text { an exponentially small error, }
\end{aligned}
$$

esp., it is enough to estimate $\int_{U \cap D} e^{ \pm}(t, x, x)$ for such a patch $U$ as described above. A close look at Levi's sum will convince the reader that $(4 \pi t)^{d / 2} \int_{U \cap D} e^{ \pm}$

$(t, x, x)$ can be developed in powers of $\sqrt{t}$. $B$ can be covered by a finite number of patches $U$ of small total volume, so terms like $t \times \operatorname{vol} U$ can be neglected: they can only influence the coefficient of $t^{3 / 2}$. As a simple application of this fact, the first term $e^{0}(t, x, x)$ of the expansion of $e^{ \pm}(t, x, x)$ contributes

$$
\begin{aligned}
(4 \pi t)^{d / 2} \int_{U \cap D} e^{0}(t, x, x)= & \int_{U \cap D} 1+\text { an error of magnitude } \\
& \leq \text { a constant multiple of } t \times \operatorname{vol} U
\end{aligned}
$$

so that, in view of $(4 a)$ and the fact that $(3.5 b)$ still holds, it suffices for the proof of (2) to check that

$$
\begin{aligned}
& (4 \pi t)^{d / 2} \int_{U \cap D} e^{0}(t, x, \dot{x}) \\
& =\frac{1}{4} \sqrt{4 \pi t} \int_{U \cap B} 1+\frac{t}{2} \int_{U \cap B} \text { flux } h+o(t \times \operatorname{vol} U), \\
& (4 \pi t)^{d / 2} \int_{U \cap D} e^{0} \sharp f(t, x, x) \\
& =-\frac{t}{6} \int_{U \cap B} \frac{\left(g^{11} \operatorname{det} g\right) .}{\operatorname{det} g} \sqrt{g_{11}}+o(t \times \operatorname{vol} U), \\
& (4 \pi t)^{d / 2} \int_{U \cap D} e^{0} \sharp f(t, x, \dot{x})=o(t \times \operatorname{vol} U) .
\end{aligned}
$$

Step $2[$ proof of $(5 \mathrm{a})]$.

(6)

$$
\begin{aligned}
(4 \pi t)^{d / 2} \int_{U \cap D} e^{0}(t, x, \dot{x}) \\
=\int_{U \cap B} d x_{2} \cdots d x_{d} \int_{0}^{1} \sqrt{\operatorname{det} g} \exp \left\{-g(\dot{x})^{1 / 2}[\dot{x}-x-b(\dot{x}) t]^{2} / 4 t\right\} d x_{1} \\
=\int_{U \cap B} d x_{2} \cdots d x_{d} \int_{0}^{1} \sqrt{\operatorname{det} g} \exp \left(-g_{11} x_{1}^{2} / t-f x_{1}-|b|^{2} t / 4\right) d x_{1}
\end{aligned}
$$


where $Q=\Delta+h \partial=a \partial^{2}+b \partial$ and $f=g_{1 k} b^{k}$; the following simplifications can be made by ignoring negligible terms:

(a) $\sqrt{\operatorname{det} g}$ can be replaced by $\sqrt{\operatorname{det} g^{0}}+(\sqrt{\operatorname{det} g})^{\cdot} x_{1}$, where $o$ stands for evaluation at $x^{0}=\left(0, x_{2}, \cdots, x_{d}\right) \in B$, since

$$
\int_{0}^{1} x_{1}^{2} d x_{1} e^{-c_{1} x_{1}^{2} / t} \leq c_{2} t^{3 / 2} .
$$

(b) $\exp \left(-g_{11} x_{1}^{2} / t-f x_{1}-|b|^{2} t / 4\right)$ can be replaced by $e^{-g_{11}^{0} x_{1}^{2} / t}\left(1-g_{11} x_{1}^{3} / t-f^{0} x_{1}\right)$ for the same reason. $\left(0 \leq e^{-x}-1+x \leq\right.$ $x^{2} / 2$ for $x \geq 0$.)

(c) $\int_{0}^{1}$ can be replaced be $\int_{0}^{\infty}$, since $\int_{1}^{\infty} e^{-c_{1} x_{1}^{2} / t} \leq \exp \left(-c_{2} / t\right)$.

After these simplifications, (6) becomes

$$
\begin{array}{r}
\int_{U \cap B} d x_{2} \cdots d x_{d} \sqrt{\operatorname{det} g^{0}} \int_{0}^{\infty} e^{-g_{11}^{0} x_{1}^{2} / t} d x_{1} \\
\times\left[1+\frac{(\sqrt{\operatorname{det} g})}{\sqrt{\operatorname{det} g^{0}}} x_{1}-g_{11} \frac{x_{1}^{3}}{t}-f^{0} x_{1}\right]
\end{array}
$$

up to a negligible error, and performing the inside integral gives

$$
\begin{aligned}
& \frac{1}{4} \sqrt{4 \pi t} \int_{U \cap B} \frac{\sqrt{\operatorname{det} g^{0}}}{\sqrt{\mathrm{g}_{11}^{0}}} d x_{2} \cdots d x_{d} \\
& \quad+\frac{t}{2} \int_{U \cap B} \frac{\sqrt{\operatorname{det} g^{0}}}{\sqrt{\mathrm{g}_{11}^{0}}} d x_{2} \cdots d x_{d} \frac{1}{\sqrt{\mathrm{g}_{11}^{0}}}\left[\frac{(\sqrt{\operatorname{det} g})}{\sqrt{\operatorname{det} g^{0}}}-\frac{g_{11}}{g_{11}^{0}}-f^{0}\right] .
\end{aligned}
$$

$f^{0}$ is now computed with the aid of (3):

$$
\begin{aligned}
f^{0}=g_{11}^{0} b_{1}^{0} & =g_{11}^{0} \frac{\left(g^{11} \sqrt{\operatorname{det} g}\right)}{\sqrt{\operatorname{det} g}}+g_{11}^{0} h^{1} \\
& =\frac{(\sqrt{\operatorname{det} g})}{\sqrt{\operatorname{det} g^{0}}}-\frac{g_{11}^{i}}{g_{11}^{0}}-\sqrt{g_{11}^{0}} \text { flux } h,
\end{aligned}
$$

and (5a) follows.

Step 3 [proof of $(5 \mathrm{~b})] .(5 \mathrm{~b})$ is not so cheap.

$$
\begin{aligned}
(4 \pi t)^{d / 2} & e^{0} \sharp f(t, x, x) \\
= & (4 \pi t)^{d / 2} \int_{0}^{t} d s \int_{R^{d}} \frac{\exp \left\{-\left|g^{1 / 2}(y)[y-x-b(y)(t-s)]\right|^{2} / 4(t-s)\right\}}{[4 \pi(t-s)]^{d / 2}} \\
\quad & \frac{\exp \left\{-\left|g^{1 / 2}(x)[x-y-b(x) s]\right|^{2} / 4 s\right\}}{(4 \pi s)^{d / 2}} \sqrt{\operatorname{det} g(y)} d y \\
\quad \times & \left\{\left[g^{i j}(y)-g^{i j}(x)\right]\right. \\
& \times\left[\frac{1}{4 s^{2}} g_{i k}(x)\left(y_{k}-x_{k}-b_{k}(x) s\right) g_{j l}(x)\left(y_{l}-x_{l}-b_{l}(x) s\right)\right. \\
& \left.\left.-\frac{g_{i j}(x)}{2 s}\right]-\left[b^{i}(y)-b^{i}(x)\right] \frac{g_{i k}(x)\left[y_{k}-x_{k}-b_{k}(x) s\right]}{2 s}\right\} .
\end{aligned}
$$


(9a) can actually be replaced by

(9b)

$$
\begin{aligned}
\int_{0}^{t} d s & \int_{R^{d}} \frac{e^{-\mid g^{1 /\left.2(x)(y-x)\right|^{2} / 4 r}}}{(4 \pi r)^{d / 2}} \sqrt{\operatorname{det} g(x)} d y \\
\times & \left\{\left[g^{i j}(y)-g^{i j}(x)\right]\left[\frac{g_{i k}(x)\left(y_{k}-x_{k}\right) g_{j l}(x)\left(y_{l}-x_{l}\right)}{4 s^{2}}-\frac{g_{i j}(x)}{4 s}\right]\right. \\
& \left.-\left[f^{i}(y)-f^{i}(x)\right] \frac{g_{i k}(x)\left(y_{k}-x_{k}\right)}{2 s}\right\}
\end{aligned}
$$

up to the desired degree of precision, where

$$
r=s(t-s) t, f^{j}=(\operatorname{det} g)^{\frac{1}{2}} \partial g^{i j}(\operatorname{det} g)^{\frac{1}{2}} / \partial x_{i}(j \leq d) .
$$

For example, to replace the first exponential in (9a) by

$$
\exp \left[-\left|g^{1 / 2}(x)(y-x)\right|^{2} / 4(t-s)\right]
$$

it suffices to note the following points:

(a) The integration over $R^{d}$ can be restricted to the figure $\mid y-$ $x \mid<(t-s)^{2 / 5}$ since, for $t \downarrow 0$, the remainder makes a contribution of magnitude smaller than

$$
\begin{gathered}
c_{1} t^{d / 2} \int_{0}^{t} d s \int_{|y-x| \geq(t-s)^{2 / 5}} d y \frac{e^{-c_{2}|y-x|^{2} /(t-s)}}{(t-s)^{d / 2}} \frac{e^{-c_{2}|y-x|^{2} / s}}{s^{d / 2}} \\
\quad \times\left(\text { terms like } s^{-2}|y-x|^{3}, s^{-1}|y-x|,\right. \text { etc., replaceable } \\
\left.\quad \text { by } c_{3} s^{-1 / 2} \text { after reducing } c_{2} \text { to } c_{4}<c_{2}\right) \\
\leq c_{5} \int_{0}^{t} \frac{d s}{\sqrt{s}} \int_{|w|>(t-s)^{2 / 5}} d w \frac{e^{-c_{4}|w|^{2} / r}}{r^{d / 2}} \\
\leq c_{6} \int_{0}^{t} \frac{d s}{\sqrt{s}} e^{-c_{7}(t-s)^{4 / 5} / r} \leq c_{8} e^{-c_{7} \sqrt[5]{t}}
\end{gathered}
$$

which is negligible.

(b) Performing the integral just over $y-x<(t-s)^{2 / 5}$ and using $e^{-A}-e^{-B} \leq(B-A) e^{-A}(0 \leq A \leq B)$ to estimate the difference between the 2 integrands, one finds that the indicated replacement produces an error of magnitude smaller than

$$
\begin{array}{rl}
C_{9} t^{d / 2} \int_{0}^{t} & d s \int_{|y-x|<(t-s)^{2 / 5}} d y \frac{e^{-c_{10}|y-x|^{2} /(t-s)}}{(t-s)^{d / 2}} \\
& \times\left[\frac{|y-x|^{3}}{t-s}+|y-x|^{2}+t-s\right] \frac{e^{-c_{10}|y-x|^{2} / s}}{s^{d / 2}} \\
& \times\left(\text { terms like } s^{-2}|y-x|^{3}, s^{-1}|y-x|, \text { etc. }\right) \\
\leq & c_{11} \int_{0}^{t} \sqrt{\frac{t-s}{s}}=c_{12} t
\end{array}
$$


which is also negligible after integrating over $U \cap D$.

(c) Finally, one makes use of the fact that for the new exponential, the integral over $|y-x|>(t-s)^{2 / 5}$ is likewise negligible.

(9b) is also to be integrated over $U \cap D$; for this purpose, similar estimates permit us to replace it by

(9c) $\int_{0}^{t} d s \int_{R^{d}} \frac{e^{-\left|g^{0} \frac{1}{2}(y-x)\right|^{2} / 4 r}}{(4 \pi r)^{d / 2}} \sqrt{\operatorname{det} g^{0}} d y$

$$
\begin{aligned}
\times\{ & {\left[\hat{g}^{i j}\left(y_{1}, x^{o}\right)-\hat{g}^{i j}(x)\right]\left[\frac{g_{i k}^{0}\left(y_{k}-x_{k}\right) g_{j l}^{0}\left(y_{\iota}-x_{\iota}\right)}{4 s^{2}}-\frac{g_{i j}^{0}}{2 s}\right] } \\
& \left.-\left[\hat{f}^{i}\left(y, x^{0}\right)-\hat{f}^{i}(x)\right] \frac{g_{i k}^{0}\left(y_{k}-x_{k}\right)}{2 s}\right\} .
\end{aligned}
$$

$\wedge$ has the following meaning: for fixed $x^{0}=\left(0, x_{2}, \cdots, x_{d}\right) \in B, \hat{g}$ is a broken line with the same corner as $g$ at $x_{1}=0$ (and no other corners), while $\hat{f}$ is a step function with a single jump at $x_{1}=0$ agreeing with $f$ at $x_{1}=0^{ \pm}$.

Do the integration $\int_{R^{d-1}} d y_{2} \cdots d y_{d}$ and use the special form of $g^{0}[(3 b)]$. This gives

$$
\begin{aligned}
& \int_{0}^{t} d s \int_{-\infty}^{+\infty} \frac{e^{g_{11}^{0}\left(y_{1}-x_{1}\right)^{2} / 4 r}}{\sqrt{4_{\pi r} / g_{11}^{0}}} d y_{1} \\
& \times\left\{[ \hat { g } ^ { i j } ( y _ { 1 } , x ^ { 0 } ) - \hat { g } ^ { i j } ( x ) ] \left[\frac{g_{i 1}^{0} g_{j 1}^{o}\left(y_{1}-x_{1}\right)^{2}}{4 r^{2}}\right.\right. \\
&\left.+\sum_{k, l \geq 2} g_{i k}^{0} g_{j l}^{0} g_{k l}^{0} \frac{2 r}{4 s^{2}}-\frac{g_{i j}^{0}}{2 s}\right] \\
&\left.-\left[\hat{f}^{i}\left(y_{1}, x^{0}\right)-\hat{f}^{i}(x)\right] \frac{g_{i 1}^{0}\left(y-x_{1}\right)}{2 s}\right\} \\
&= \int_{0}^{t} d s \int_{R^{1}} \frac{e^{-g_{11}^{0}\left(y_{1}-x_{1}\right)^{2} / 4 r}}{\sqrt{4 \pi r / g_{11}^{0}}} d y_{1} \\
& \times\left\{\left[\hat{g}^{11}\left(y_{1}, x^{0}\right)-\hat{g}^{11}(x)\right]\left[\frac{\left(g_{11}^{0}\right)^{2}\left(y_{1}-x_{1}\right)^{2}}{4 s^{2}}-\frac{g_{01}^{1}}{2 s}\right]\right. \\
&-\sum_{i, j \neq 1}\left[\hat{g}^{i j}\left(y_{1}, x^{0}\right)-\hat{g}^{i j}(x)\right] \frac{g_{i j}^{0}}{2 t} \\
&\left.-\left[\hat{f}^{1}\left(y_{1}, x^{0}\right)-\hat{f}^{1}(x)\right] \frac{g_{11}^{0}}{2 s}\left(y_{1}-x_{1}\right)\right\} .
\end{aligned}
$$


CURVATURE AND EIGENVALUES

59

(aa) implies that for $i=j=1$ or $i, j \geq 2, \hat{g}^{i j}\left(y_{1}, x^{0}\right)-\hat{g}^{i j}(x)=$ $\left(y_{1}-x_{1}\right)^{i j .}$ or $-\left(y_{1}+x_{1}\right) g^{i j}$. according as $y_{1}>0$ or $y_{1}>0$; also $\hat{f}^{1}\left(y_{1}, x^{0}\right)-\hat{f}^{1}(x)=0$ or $-2\left(\operatorname{det} g^{0}\right)^{-1 / 2}\left(g^{11} \sqrt{\operatorname{det}} g\right) \cdot$ according as $y_{1}>$ 0 or $y_{1},<0, g^{11} \sqrt{\operatorname{det} g}$ being even across $B$, so (10a) simplifies to

$(10 \mathrm{~b})$

$$
\begin{aligned}
\int_{0}^{t} d s & \int_{-\infty}^{-x_{1}} \frac{e^{-g_{11}^{0} \omega_{1}^{2} / 4 r}}{\sqrt{4 \pi r / g_{11}^{0}}} d w_{1} \\
\quad \times & \left\{-2\left(x_{1}+w_{1}\right) g^{11} \cdot\left[\frac{\left(g_{11}^{0}\right)^{2} w_{1}^{2}}{4 s^{2}}-\frac{g_{11}^{0}}{2 s}\right]\right. \\
& \left.+2\left(x_{1}+w_{1}\right) \sum_{i, j \neq 1} g^{i j \cdot} \frac{g_{i j}^{0}}{2 t}+\frac{\left(g^{11} \sqrt{\operatorname{det} g)}\right.}{\sqrt{\operatorname{det} g^{0}}} \frac{g_{11}^{0}}{s} w_{1}\right\}
\end{aligned}
$$

Do next the integral $\int_{0}^{1}(10 \mathrm{~b}) \sqrt{\operatorname{det} g} d x_{1}$, replacing $g$ by $g^{0}$ extending the integration from 1 to $+\infty$, and changing

$$
\int_{0}^{\infty} d x_{1} \int_{-\infty}^{-x_{1}} d w_{1} \text { into } \int_{-\infty}^{0} d w_{1} \int_{0}^{-w_{1}} d x_{1}
$$

(11)

$$
\begin{aligned}
& \sqrt{\operatorname{det} g^{0}} \int_{0}^{t} d s \int_{-\infty}^{0} \frac{e^{-g_{11}^{0} w_{1}^{2} / 4 r}}{\sqrt{4 \pi r / g_{11}^{0}}} d w_{1} \\
& \times\left[\frac{w_{1}^{2}}{2} g^{11} \cdot\left(\frac{\left(g_{11}^{0}\right)^{2} w_{1}^{2}}{4 s^{2}}-\frac{g_{11}^{0}}{2 s}\right)\right. \\
& \left.-\sum_{i, j \neq 1} g^{i j \cdot} g_{i j}^{0} \frac{w_{1}^{2}}{4 t}-\frac{\left(g^{11} \sqrt{\operatorname{det} g)}\right.}{\sqrt{\operatorname{det}} g^{0}} g_{11}^{0} \frac{w_{1}^{2}}{s}\right] \\
& =\sqrt{\operatorname{det} g^{0}} \int_{0}^{t} d s\left\{\frac{g^{11} \cdot}{2}\left[3 \frac{(t-s)^{2}}{t^{2}}-\frac{t-s}{t}\right]\right. \\
& \left.-\frac{1}{2} \sum_{i, j \neq 1} g^{i j} \cdot g_{i j}^{0} g_{11}^{0}-1 \frac{s(t-s)}{t^{2}}-\frac{\left(g^{11} \sqrt{\operatorname{det} g)^{\cdot}}\right.}{\sqrt{\operatorname{det} g^{0}}} \frac{t-s}{t}\right\} \\
& =t \sqrt{\operatorname{det} g^{0}} \times\left[\frac{g^{11} \cdot}{4}-\frac{1}{12} \sum_{i, j \neq l} g^{i j} \cdot g_{i j}^{0} g_{11}^{0-1}-\frac{1}{2} \frac{\left(g^{11} \sqrt{\operatorname{det} g}\right)^{-}}{\sqrt{\operatorname{det} g^{0}}}\right] \\
& =-\frac{t}{6} \sqrt{\operatorname{det} g^{0}} \frac{\left(g^{11} \operatorname{det} g\right)}{\operatorname{det} g^{0}} \text {, }
\end{aligned}
$$


since $g^{i j} \cdot g_{i j}=-(\operatorname{det} g) \cdot \sqrt{\operatorname{det} g}$. An integration $\int_{\cup \cap{ }_{B}}(11) d x_{2} \cdots d x_{d}$ now gives the desired formula $(5 b)$.

Step 4 . The proof of $(5 \mathrm{c})$ is practically the same, so it is left to the industrious reader.

\section{6. $\Delta$ on differential forms}

Given a closed manifold $M$, let $\Delta$ act on the space $\Lambda^{p}$ of smooth exterior differential $p$-forms $(p \leq d) . \Lambda^{p}$ is a pre-Hilbert space relative to the inner product $\left(f_{1}, f_{2}\right)=\int<f_{1}, f_{2}>,<f_{1}, f_{2}>$ being the Riemannian inner product of $p$-forms at a point of $M$, and $\Delta$ can be expressed as $-\left(d d^{*}+d^{*} d\right), d: \Lambda^{p-1} \rightarrow \Lambda^{p}(1 \leq p \leq d)$ being the exterior differential and $d^{*}: \Lambda^{p+1} \rightarrow \Lambda^{p}(0 \leq p<d)$ its dual relative to the above inner product. $\Delta$ acting on $\Lambda^{p}$ is symmetric with a discrete spectrum:

$$
0 \geq \gamma_{0} \geq \gamma_{1} \geq \gamma_{2} \geq \text { etc. } \quad \downarrow-\infty
$$

the corresponding eigenforms $f$ form a unit perpendicular basis of $\Lambda^{p}$, the sum

$$
e^{p}=\sum_{n \geq 0} \exp \left(\gamma_{n} t\right) f_{n} \otimes f_{n}
$$

converges uniformly on compact figures of $(0, \infty) \times M^{2}$ to the elementary solution of $\partial u / \partial t=\Delta u$ for $p$-forms and the spur $Z^{p}=\sum \exp \left(\gamma_{n} t\right)$ of $e^{t \Delta}$ on $\Lambda^{p}$ can be expressed [14] as the integral over the manifold of the pole sp $e^{p}=\sum \exp \left(\gamma_{n} t\right)<f_{n}, f_{n}>: Z^{p}=\int \mathrm{sp} e_{p}$.

Define $Z$ to be the alternating sum of $Z^{p}(p \leq d): Z=Z^{0}-Z^{1}+$ $\cdots \pm Z^{d}$. Then

$$
Z=\text { the Euler Characteristic } E \text { of } M \text {, }
$$

as will be proved below. Poincaré duality makes this trivial for odd dimensions $\left(Z^{p}=Z^{d-p}\right)$; also, in 2 dimensions $Z^{0}=Z^{2}$ for the same reason, so from (1.5b) and (1) it follows that for $d=2$,

$$
Z^{1}=2 Z^{0}-E=\frac{\text { area }}{2 \pi t}-\frac{2}{3} E+\frac{\pi t}{30} \int K^{2}+o\left(t^{2}\right) .
$$

Given a number $\gamma \leq 0$, define $3^{p}$ to be the eigenspace of $p$-forms $f$ such that $\Delta f=\gamma f$. By de Rham's theorem [14],

$$
\operatorname{dim} 3^{0}-\operatorname{dim} 3^{1}+\cdots \pm \operatorname{dim} 3^{d}=E \quad \text { for } \quad \gamma=0
$$

so (1) is the same as

$$
\operatorname{dim} 3^{0}-\operatorname{dim} 3^{1}+\cdots \pm \operatorname{dim} 3^{d}=0 \quad \text { for } \quad \gamma<0
$$


Chern [4] discovered a beautiful extension of the classical GaussBonnet formula to manifolds of even dimension $d>2$. Chern's formula states that $\int C=E$. $C$ is a (complicated) homogeneous polynomial of degree $d / 2$ in the entries of the curvature tensor, reducing to the classical integrand $K / 2 \pi=-R_{12}^{12} / 2 \pi$ for $d=2$. Because of the complete cancellation of the time-dependent part of the alternating sum $Z$, it is natural to hope that some fantastic cancellation will also take place in the small, i.e., in the alternating pole sum:

$$
\operatorname{sp~}^{0}-\mathrm{sp}^{1}+\cdots \pm \mathrm{sp} e^{d}=\left\{\begin{array}{l}
0 \\
+o(1) \quad \text { for } \quad d\left\{\begin{array}{l}
\text { odd } \\
\mathrm{C}
\end{array}\right. \text { even }
\end{array}\right.
$$

Poincaré duality does it for odd $d$ with $o(1)=0$, but the even-dimensional proof eludes us, except for $d=2$; in which case

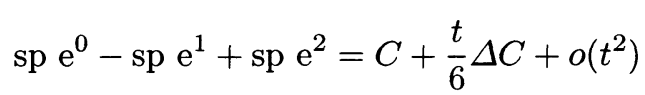

(see [8] for additional information for $d=4$ ). The proof of (5) is postponed until after the

Proof of $(1)=(3 \mathrm{~b})$. Choose $\gamma<0$, let $3^{p}(p \leq d)$ be the corresponding eigenspaces, and make the convention that $3^{-1}=3^{d+1}=0 . \Delta=$ $-\left(d^{*} d+d d^{*}\right)$ commutes with $d$ and $d^{*}$, so $d 3^{p-1}+d^{*} 3^{p+1} \subset 3^{p}$. Because $d^{2} \quad=\quad 0,\left(d 3^{p-1}\right.$, $\left.d^{*} 3^{p+1}\right)=\left(d^{2} 3^{p-1}, 3^{p+1}\right)=0$, so the sum is direct, and it fills up the whole of $3^{p}\left(=d 3^{p-1} \oplus d^{*} 3^{p+1}\right)$ since, for $f \subset 3^{p}$,

$$
\left(f, d 3^{p-1}\right)=\left(d^{*} f, 3^{p-1}\right)=0, \quad\left(f, d^{*} 3^{p+1}\right)=\left(d f, 3^{p+1}\right)=0
$$

make $d^{*} f=d f=0$, so that $\gamma f=\Delta f=0$ and $f=0(\gamma \neq 0)$; esp.,

$$
\operatorname{dim} 3^{p}=\operatorname{dim} d 3^{p-1}+\operatorname{dim} d^{*} 3^{p+1} \quad(p \leq d),
$$

and so

$$
\sum_{p \leq d}(-1)^{p} \operatorname{dim} 3^{p}=\sum\left(-\operatorname{dim} d^{*} 3^{2 p}+\operatorname{dim} 3^{2 p}-\operatorname{dim} d 3^{2 p}\right) .
$$

But $3^{2 p}=d 3^{2 p-1} \oplus d^{*} 3^{2 p+1}$, so that

$\operatorname{dim} 3^{2 p}-\operatorname{dim} d^{*} 3^{2 p}-\operatorname{dim} d 3^{2 p}$

$$
=\operatorname{dim} d 3^{2 p-1}+\operatorname{dim} d^{*} 3^{2 p+1}-\operatorname{dim} d^{*} d 3^{2 p-1}-\operatorname{dim} d d^{*} 3^{2 p+1} \geq 0,
$$

and also

$$
\begin{aligned}
\operatorname{dim} & 3^{2 p}-\operatorname{dim} d^{*} 3^{2 p}-\operatorname{dim} d 3^{2 p} \\
& =\operatorname{dim} \Delta 3^{2 p}-\operatorname{dim} d^{*} 3^{2 p}-\operatorname{dim} d 3^{2 p} \\
& \leq \operatorname{dim} d d^{*} 3^{2 p}+\operatorname{dim} d^{*} d 3^{2 p}-\operatorname{dim} d^{*} 3^{2 p}-\operatorname{dim} d 3^{2 p} \leq 0
\end{aligned}
$$


in brief, $\operatorname{dim} 3^{2 p}=\operatorname{dim} d 3^{2 p}+\operatorname{dim} d^{*} 3^{2 p}$, and the whole of the alternating dimension sum (6) collapses to 0 .

Proof of (5) $(d=2) .3^{1}=d 3^{0} \oplus d^{*} 3^{2}$ for $\gamma<0$, and for $f \in 3^{0}$,

$$
\|d f\|^{2} \equiv(d f, d f)=-\left(d^{*} d f, f\right)=-(\Delta f, f)=-\gamma\|f\|^{2}
$$

with a similar result $\left(\left\|d^{*} f\right\|^{2}=-\gamma\|f\|^{2}\right)$ for $f \in 3^{2}$. Because of this,

$$
\begin{aligned}
\sum \exp \left(\gamma_{n}^{0} t\right) & <d f_{n}^{0}, d f_{n}^{0}>+\sum \exp \left(\gamma_{n}^{2} t\right)<d^{*} f_{n}^{2}, d * f_{n}^{2}> \\
& =-\sum \gamma_{n}^{1} \exp \left(\gamma_{n}^{1} t\right)<f_{n}^{1}, f_{n}^{1}>
\end{aligned}
$$

with a self-evident notation. But, for $f \in \Lambda^{0}$,

$$
<d f, d f>=g^{i j} \frac{\partial f}{\partial x_{i}} \frac{\partial f}{\partial x_{j}}=\frac{1}{2} \Delta f^{2}-f \Delta f
$$

so, by the Poincaré duality between $3^{0}$ and $3^{2}$,

$$
\begin{aligned}
& -\frac{\partial}{\partial t} \mathrm{sp}^{1}=-\sum \gamma_{n}^{1} \exp \left(\gamma_{n}^{1} t\right)<f_{n}^{1}, f_{n}^{1}>=2 \sum \exp \left(\gamma_{n}^{0} t\right)<d f_{n}^{0}, d f_{n}^{0}> \\
& =\sum \exp \left(\gamma_{n}^{0} t\right)\left[\Delta\left(f_{n}^{0}\right)^{2}-2 f_{n}^{0} \Delta f_{n}^{0}\right]=\Delta \mathrm{sp} \mathrm{e}^{0}-2 \frac{\partial}{\partial t} \operatorname{sp} e^{0}
\end{aligned}
$$

or, what is the same,

$$
\frac{\partial}{\partial t}\left(\mathrm{sp} \mathrm{e}^{0}-\mathrm{sp}^{1}+\mathrm{sp}^{2}\right)=\Delta \mathrm{sp} \mathrm{e}^{0}
$$

sp $\mathrm{e}^{0}$ has an expansion beginning with a multiple of $t^{-1}$ and proceeding by ascending powers of $t$ as stated in $\S 4$, and a little extra attention to the proof shows that the formal application of $\Delta$ to this expansion gives the expansion for $\Delta \mathrm{sp} \mathrm{e}^{0}$. Consequently, (4.1) implies

$$
\mathrm{sp} \mathrm{e}^{0}-\mathrm{sp} \mathrm{e}^{1}+\mathrm{sp}^{2}=B+\frac{t}{6} \Delta C+o\left(t^{2}\right)
$$

with $C=$ the Gauss-Bonnet integrand $K / 2 \pi$, and to complete the proof of (5), it remains to check that $B=C$. Pick local coordinates so that Cartan's formula (4.4) holds. A moment's reflection shows that $B$ can be expressed as a (universal) combination of second partials of $g_{i j}(i, j \leq 2)$; as such, it is a (universal) constant multiple of the one nonzero component $R_{1212}$ of the Riemann tensor, and the constant can be identified as $-1 / 2 \pi$ by using the Gauss-Bonnet formula in the special case of the Riemann sphere:

$$
\begin{aligned}
2 & =E=\int\left(\mathrm{sp} \mathrm{e}^{0}-\mathrm{sp}^{1}+\mathrm{sp}^{2}\right)=B \\
& =\text { constant } \times \int R_{1212}=- \text { constant } \times 4 \pi .
\end{aligned}
$$




\section{Algebraic computation of $k_{1}$ and $k_{2}$}

The style of proof just used to finish the verification of (2.5) will now be exploited to compute the third coefficient of the Minakshisundaram expansion (4.3) for $Q=\Delta$ :

$$
k_{2}=\frac{1}{180}(10 A-B+2 C)+\text { constant } \times \Delta K,
$$

with

$$
\begin{gathered}
A=\left(\sum_{i<j} R_{i j i j}\right)^{2}=K^{2}, \\
B=\sum_{j, k}\left(\sum_{i} R_{i j i k}\right)^{2}, \\
C=\sum_{i, j, k, l}\left(R_{i j k l}\right)^{2} .
\end{gathered}
$$

The constant multiplier of $\Delta K$ in (1) is not known, but $\int_{M} \Delta K=0$, so

$$
\int_{M} k_{2}=\frac{1}{180} \int_{M}(10 A-B+2 C)
$$

as needed for (1. 5a); in any case, this constant is universal, i.e., it is the same for all manifolds $M$. The method will also provide us with a new derivation of the formula $k_{1}=K / 3$. A short table of special expansions will be helpful for the proof; in this table $Z$ is computed up to an exponentially small error for several standard manifolds. $D^{2}\left(D^{3}\right)$ is the 2(3)-dimensional Lobachevsky space modulo a discontinuous group of motions.

Pick exponential coordinates on a patch about a point $o \in M$ as for (4.4). The coefficients of the power series expansion of $g$ about $o$ will be polynomials in the curvature tensor $R$ and its covariant derivatives [3, Chapter 10, §4], and it follows from this and from Levi's sum for the pole of $e$ that the coefficients of (4.3) are expressible as polynomials of the same kind. A scaling argument now gives the degree of these polynomials. Change $g$ into $C^{2} g\left(C^{2}>0\right)$. Then $\Delta$ is changed into $C^{-2} \Delta$, and the pole of the elementary solution becomes $e\left(t / C^{2}, o, o\right) C^{-d}$, so that $k_{n}$ is simply multiplied by $C^{2 n}$. But also, an $l$-fold covariant derivative of $R\left(C^{2} g\right)$ is a multiple of $C^{2+l}$. Consequently, $k_{n}=k_{n}(g)$ is a "homogeneous polynomial" of degree $2 n$ in $R$ and its covariant derivatives, if

\begin{tabular}{c|c|c|c|c|l}
\hline \multicolumn{1}{|c}{ TABLE } \\
\hline$M$ & $K$ & $A$ & $B$ & $C$ & $Z /(4 \pi t)^{d / 2} \times \operatorname{vol} M$ \\
\hline$S^{2}$ & 1 & 1 & 2 & 2 & $\frac{e^{t / 4}}{\sqrt{\pi t}} \int_{0}^{1} \frac{e^{-x / t}}{\sin \sqrt{x}} d x=1+\frac{t}{3}+\frac{t^{2}}{15}+\cdots$ \\
\hline$S^{3}$ & 3 & 9 & 12 & 6 & $e^{t}=1+t+2 t^{2}+\cdots$ \\
\hline$D^{2}$ & -1 & 1 & 2 & 2 & $\frac{e^{-t / 4}}{\sqrt{\pi t}} \int_{0}^{1} \frac{e^{-x / t}}{\sin h \sqrt{x}} d x=1+\frac{t}{3}+\frac{t^{2}}{15}+\cdots$ \\
\hline$D^{3}$ & -3 & 9 & 12 & 6 & $e^{-t}=1-t+\frac{1}{2} t^{2}+\cdots$ \\
\hline
\end{tabular}


to an $l$-fold covariant derivative is ascribed the degree $2+l$, esp., $k_{1}$ is a form of degree 1 in $\mathrm{R}$, while $k_{2}$ is a form of degree 2 in $R$ plus a form of degree 1 in second covariant derivatives of $R$. Clearly, the coefficients of these forms depend upon $M$ only via the dimension.

The next step is to exploit the fact that an orthogonal transformation of the tangent space changes one exponential coordinate system $x$ into another. Because the pole of $e$ depends on $x$ only via $\sqrt{ } \operatorname{det} g$, which is an orthogonal invariant, the coefficients of its expansion are likewise orthogonal invariants, esp., $k_{1}$ is an invariant form of degree 1 in $R$, and as such, it is a constant multiple of $K=-\sum_{i<j} R_{i j i j}[19$,

Chapter 5]. This constant depends upon the dimension of $M$ only, so to complete the evaluation of $k_{1}$, it suffices to check that the constant is dimension-free and to compute it for $M=S^{2}$, say (see the TABLE). To settle the first point, look at a product manifold, $M=M_{1} \times M_{2}$. $\Delta(M)=\Delta\left(M_{1}\right) \otimes 1 \oplus 1 \otimes \Delta\left(M_{2}\right)$, so $\mathrm{e}(M)=e\left(M_{1}\right) \otimes e\left(M_{2}\right)$, and it follows from (4.3) that $k_{1}(M)=k_{1}\left(M_{1}\right)+k_{1}\left(M_{2}\right)$. But also $R(M)=$ $R\left(M_{1}\right) \oplus R\left(M_{2}\right)$, so that $K(M)=K\left(M_{1}\right)+K\left(M_{2}\right)$, and varying the dimension of $M_{2}$ leads at once to the proof.

$k_{2}$ is not so simple.

Step 1 is to notice that the forms of degrees 2 and 1 into which $k_{2}$ is split are separately invariant under the action of the orthogonal group. As stated before, the coefficients of these forms depend upon dimension only.

Step 2. For $d>3$, the space of curvature tensors at a point of $M$, viewed as a representation space of the orthogonal group $0(d)$, splits into 3 irreducible pieces. One piece is the kernel of the contraction map $R_{i j k l} \rightarrow R_{i j i l}$. The orthogonal complement can be viewed as the space of symmetric matrices with $0(d)$ acting by similarity $\left(x \rightarrow 0^{*} x o\right)$, and this piece splits into the scalars plus symmetric matrices with spur 0 [19, Chap. 5]. Consequently, the space of invariant polynomials of degree 2 is 3 -dimensional, the 3 polynomials $A, B, C$ exhibited in (2) provide us with a nice basis, and the corresponding part of $k_{2}$ is simply $c_{0} A+c_{1} B+c_{2} C$ with coefficients depending (perhaps) on the dimension. The same still holds for dimensions 2 and 3, except that

$$
\begin{array}{ll}
B=C=2 A & (d=2), \\
B=A+C / 2 & (d=3),
\end{array}
$$

which make the splitting simpler. 
Step 3. The part of $k_{2}$ which is an invariant form of degree 1 in second covariant derivatives of $R$ can only be obtained by a 3 -fold contraction [19, Chap. 5], and only 2 candidates present themselves: $R_{i j i j ; k k}=-2 \Delta K$ and $R_{i k j k ; i j}$. But, by the Bianchi identities,

$$
R_{i k j k ; i j}+R_{i k k i ; j j}+R_{i k i j ; k j}=0
$$

so the second candidate is half the first, and

$$
k_{2}=c_{0} A+c_{1} B+c_{2} C+c_{3} \Delta K
$$

with coefficients depending upon dimension only.

Step 4 is to prove that the coefficients are dimension-free. This is done, as in the proof of $k_{1}=K / 3$, by looking at a product $M=$ $M_{1} \times M_{2} \cdot R(M)=R\left(M_{1}\right) \oplus R\left(M_{2}\right)$, so

$$
\begin{aligned}
& A(M)=A\left(M_{1}\right)+A\left(M_{2}\right)+2 K\left(M_{1}\right) K\left(M_{2}\right) \\
& B(M)=B\left(M_{1}\right)+B\left(M_{2}\right) \\
& C(M)=C\left(M_{1}\right)+C\left(M_{2}\right)
\end{aligned}
$$

also

$$
e(M)=e\left(M_{1}\right) \otimes e\left(M_{2}\right)
$$

and a comparison of the expansion

$$
\begin{aligned}
& 1+t k_{1}(M)+t^{2} k_{2}(M)+0\left(t^{3}\right)=1+\frac{t}{3}\left[K\left(M_{1}\right)+K\left(M_{2}\right)\right] \\
& +t^{2} \times\left\{c_{0}(d)\left[A\left(M_{1}\right)+A\left(M_{2}\right)+2 K\left(M_{1}\right) K\left(M_{2}\right)\right]\right. \\
& +c_{1}(d)\left[B\left(M_{1}\right)+B\left(M_{2}\right)\right]+c_{2}(d)\left[C\left(M_{1}\right)+C\left(M_{2}\right)\right] \\
& \left.+c_{3}(d)\left[\Delta K\left(M_{1}\right)+\Delta K\left(M_{2}\right)\right]\right\}+0\left(t^{3}\right)
\end{aligned}
$$

$d$ being $\operatorname{dim} \mathrm{M}$, with the expansion

$$
\begin{aligned}
{[1+} & \left.t k_{1}\left(M_{1}\right)+t^{2} k_{2}\left(M_{1}\right)\right]\left[1+t k_{1}\left(M_{2}\right)+t^{2} k_{2}\left(M_{2}\right)\right]+o\left(t^{3}\right) \\
= & 1+\frac{t}{3}\left[K\left(M_{1}\right)+K\left(M_{2}\right)\right]+t^{2} \times\left[\frac{1}{9} K\left(M_{1}\right) K\left(M_{2}\right)\right. \\
& +c_{0}\left(d_{1}\right) A\left(M_{1}\right)+c_{1}\left(d_{1}\right) B\left(M_{1}\right)+c_{2}\left(d_{1}\right) C\left(M_{1}\right)+c_{3}\left(d_{1}\right) \Delta K\left(M_{1}\right) \\
& \left.+c_{0}\left(d_{2}\right) A\left(M_{2}\right)+c_{1}\left(d_{2}\right) B\left(M_{2}\right)+c_{2}\left(d_{2}\right) C\left(M_{2}\right)+c_{3}\left(d_{2}\right) \Delta K\left(M_{2}\right)\right] \\
& +o\left(t^{3}\right)
\end{aligned}
$$

in case $M_{1}$ is a flat torus $\left[R\left(M_{1}\right)=0\right]$ shows that the expression 


$$
c_{0}(d) A\left(M_{2}\right)+c_{1}(d) B\left(M_{2}\right)+C_{2}(d) C\left(M_{2}\right)+C_{3}(d) \Delta K\left(M_{2}\right)
$$

is independent of $d \geq d_{2}$. The fact that the coefficients are dimensionfree for $d \geq 4$ is immediate from this. For $d \leq 3$, the coefficients can be chosen to be the same as for higher dimensions.

Step 5 is to compute the actual values of the coefficients. Comparison of the terms involving $K\left(M_{1}\right) K\left(M_{3}\right)$ in (7a) and (7b) gives

$$
c_{0}=1 / 18
$$

and, from the TABLE placed at the beginning of this section,

$$
\begin{aligned}
& c_{1}=-1 / 180, \\
& c_{2}=1 / 90
\end{aligned}
$$

so that only $c_{3}$ is still unknown. This completes the proof.

For $d=4$, the integrand for Chern's extension of the Gauss-Bonnet formula [5] is easily evaluated as $\left(8 \pi^{2}\right)^{-1}(A-B+C / 2)$. The formula states that this integrates to the Euler characteristic $E$ of $M$, whence, for $d=4$,

$$
\int k_{2}=\frac{2 \pi^{2}}{45} E+\frac{1}{180} \int(9 A+3 C / 2) \geq \frac{2 \pi_{2}}{45} E
$$

$$
M \text { is a flat space if } \int k_{2}=0 \text { and } E \geq 0
$$

(10d) if the sectional curvatures of $M$ do not change sign, then

$$
\int k_{2}=0 \text { only for a flat space, }
$$

while, for $d \leq 3$,

$$
\int k_{2} \geq 0 \text { and } \int k_{2}=0 \text { only for a flat space. }
$$

Proof. (10a) is immediate from Chern's formula and (10b) follows, since $\int C=0$ makes $M$ flat. $E \geq 0$ if $M$ is simply connected. But a flat compact space is not simply connected, so (10c) is proved. (10d) is proved in the same way using the fact that $E \geq 0$ if the sectional curvatures of $M$ do not change sign [5]. The proof of (10e) is immediate from (1) and (4).

The computation of $k_{3}, k_{4}$, etc. is a problem of classical invariant theory; see for instance [17]. It looks pretty hopeless. 


\section{Open problems}

$1^{\circ}$. For $Q=\Delta$, compute all the coefficients of Minakshisundaran's expansion (4.3) and explain the geometrical significance of each. It is an open problem to find the corresponding corrections to Weyl's formula (1.2). But notice that even for $M=S^{2},-\gamma_{n}$ does not behave like $c_{-1} n+c_{0}+c_{1} n^{-1}+$ etc..

$2^{\circ}$. Prove or disprove (6.4) for even $d \geq 4$; see (7. 10a) for partial in formation in case $d=4$.

$3^{\circ}$. J. Milnor [8] proved that the spectrum of $\Delta$ acting on the differential forms of a closed manifold $M$ is not sensitive enough to discriminate between the possible Riemannian geometries on $M$. Milnor's example depends upon an example of E. Witt of 2 self-dual 16dimensional lattices $\Gamma$, dissimilar under the action of $0(16)$, but with $\sharp(R)=\sharp(\omega \in \Gamma:|\omega| \leq R)$ the same for both. Because the lattices are dissimilar, the tori $M=R^{16} / \Gamma$ are not isometric. But the spectrum of $\Delta$ on functions is just the numbers $4 \pi^{2}|\omega|^{2}$ with $\omega$ from $\Gamma$. Because $\Delta\left(f d x_{i_{1}} \wedge \cdots \wedge d x_{i p}\right)=(\Delta f) d x_{i_{1}} \wedge \cdots \wedge d x_{i p}$, the spectrum of $\Delta$ on $p-$ forms is the same, but just repeated $16 ! / p !(16-p)$ ! times, so that the 2 tori are identical from the spectral point of view. Despite this example, it may be possible to "hear" the geometry of $M$ for small dimensions ( $d=2$, for instance) or for a special class of manifolds (topological spheres, for instance). Kac [6] has asked if the spectra of both $\Delta$ for a flat plane region $D$ suffice to determine $D$ up to a rigid motion of $k_{2}$; his conjecture is no. If that is so then probably the complete geometry of a closed manifold cannot be heard even for $d=2$ and $M$ a topological sphere. But it should be noted that for $D=(0,1), 0<f \in C[0,1]$, and $Q u=f u^{\prime \prime}, f$ can be recovered from the spectra of $Q^{b}[2]$.

$4^{\circ}$. Jacobi's transformation of the theta-function shows that for $\Delta$ acting on functions on a flat torus $M=R^{d} / \Gamma$,

$$
\begin{aligned}
Z & =\sum e^{-4 \pi^{2}|\omega|^{2} t}=\frac{\operatorname{vol} M}{(4 \pi t)^{d / 2}} \sum_{\omega \in l} e^{-|\omega|^{2} / 4 t} \\
& =\frac{\operatorname{vol} M}{(4 \pi t)^{d / 2}}+\text { an exponentially small error }
\end{aligned}
$$

where $\Gamma^{*}$ is the dual lattice of $\Gamma$. Does there exist a Jacobi like transformation of $Z$ for any other manifolds? To our knowledge the only similar thing is the so-called Kramers-Wannier duality for the 2-dimensional ISING model of statistical mechanics. Both Kramers-Wannier and Jacobi's transformation are instances of Poisson's summation formula [7]. Perhaps Selberg's trace formula could be helpful in this. A simple case to look at would be a compact symmetric space $M=G / K$ of rank 1 , since the pole sp $\mathrm{e}^{0}$ is constant on $M$ and can be computed using just the radial part $A^{-1} \frac{\partial}{\partial R} A \frac{\partial}{\partial R}$ of $\Delta(A=$ the area of the spherical surface of radius $R$ about the north pole). A second interesting case would be that of a closed Riemann surface of genus $\geq 2$, viewed as the open unit disc modulo a discontinuous group. One may conjecture that the breaking off the expansion of $Z$ at the first (volume) term happens for fiat spaces only [see (7. 10) for the proof in case $d \leq 3$ and for partial information in case $d=4]$. 
$5^{\circ}$. A Jacobi transformation for $Z$ goes over into a Riemann like identity for the zeta-like function $\sum_{n \geq 1}\left|\gamma_{n}\right|^{-s}$ via the transformation

$$
Z \rightarrow \Gamma(s)^{-1} \int_{0}^{\infty} t^{s-1}(Z-1) d t .
$$

Minakshisundaram [9] used (4. 3) to prove that this zeta-function is meromorphic in the whole $s$-plane; see [11] for additional information. Expanding $Z$ as $c_{0} t^{-d / 2}+c_{1} t^{-d / 2+1}+$ etc., one finds that the zetafunction has simple poles with residues $c_{n}$ at the places $d / 2-n(n \geq 0)$ if $d$ is odd, $(0 \leq n<d / 2)$ if $d$ is even. For even $d$, the value of the zeta-function at $s=0$ is $c_{d / 2}=\int k_{d / 2}$, so that contact is made with $R$. Seeley's computation of this number [15] and with $2^{\circ}$.

\section{Bibliography}

[1] M. Berger, Sur les spectre d'une variété riemannienne, C. R. Acad Sci. Paris 263 (1966) 13-16.

[2] G. Borg, Eine Umkehrung der Sturm-Liouvilleschen Eigenwertaufgabe Bestimmung der Differential Gleichung durch die Eigenwerte, Acta Math. 78 (1946) 1-96.

[3] É. Cartan, Leçons sur la géométrie des espaces de Riemann, Gauthier-Villars, Paris, 1928.

[4] S. S. Chern, A simple intrinsic proof of the Gauss-Bonnet formula for closed Riemannian manifolds, Ann. of Math. 45 (1944) 747752 .

[5] - On the curvature and characteristic classes of a Riemannian manifold, Abh. Math. Sem. Univ. Hamburg 20 (1956) 117-126.

[6] M. Kac, Can one hear the shape of a drum? Amer. Math. Monthly 73 (April, 1966) 1-23.

[7] H. P. McKean, Jr., Kramers-Wannier duality as an instance of the Poisson summation formula, J. Math. Phys. 5 (1964) 775-776.

[8] J. Milnor, Eigenvalues of the Laplace operator on certain manifolds, Proc. Nat. Acad. Sci. U.S.A. 51 (1964) 542.

[9] S. Minakshisundaram, A generalization of Epstein zeta functions, Can. J. Math. 1 (1949) 320-329.

[10] — Eigenfunctions on Riemannian manifolds, J. Indian Math. Soc. 17 (1953) 158-165.

[11] S. Minakshisundaram \& $\dot{A}$. Pleijel, Some properties of the eigenfunctions of the Laplace operator on Riemannian manifolds, Can. J. Math. 1 (1949) 242-256.

[12] E. Nelson, The adjoint Markov process, Duke Math. J. 25 (1958) 671-690. 
[13] A. Pleijel, A study of certain Green's functions with applications in the theory of vibrating membranes, Ark. Mat. 2 (1954) 553-569.

[14] G. de Rham, Varieties differentiables, Hermann, Paris, 1960.

[15] R. Seeley, The power $\mathrm{A}^{\mathrm{s}}$ of an elliptic operator A, to appear in Proc. Sympos. on Singular Integrals, Chicago.

[16] S. Varadhan, Diffusion processes with a small parameter, to appear in Comm. Pure Appl. Math.

[17] R. Weitzenböck, Invariantentheorie, P. Noordhoff, Groningen, 1923.

[18] H. Weyl, Das asymptotische Verteilungsgesetz der Eigenschwingungen eines beliebig gestalteten elastischen Körpers. Rend. Cir. Mat. Palermo 39 (1950) 1-50.

[19] — The classical groups, Princeton University Press, Princeton, 1946.

ROCKEFEller UNIVERSITY, NeW YORK Massachusetts Institute of TEChNOLOGY 
